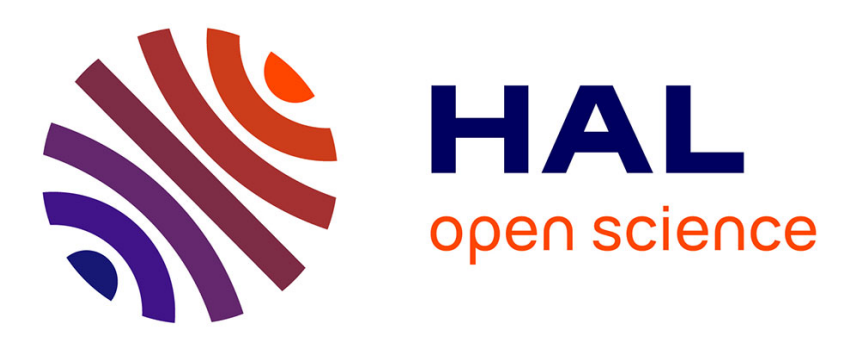

\title{
How to gain insight into the polydispersity of tannins: A combined MS and LC study
}

Laetitia Mouls, Virginie Hugouvieux, Jean Paul Mazauric, Nicolas Sommerer, Gerard Mazerolles, Helene Fulcrand

\section{- To cite this version:}

Laetitia Mouls, Virginie Hugouvieux, Jean Paul Mazauric, Nicolas Sommerer, Gerard Mazerolles, et al.. How to gain insight into the polydispersity of tannins: A combined MS and LC study. Food Chemistry, 2014, 165, pp.348-353. 10.1016/j.foodchem.2014.05.121 . hal-01837705

\section{HAL Id: hal-01837705 https://hal.science/hal-01837705}

Submitted on 27 May 2020

HAL is a multi-disciplinary open access archive for the deposit and dissemination of scientific research documents, whether they are published or not. The documents may come from teaching and research institutions in France or abroad, or from public or private research centers.
L'archive ouverte pluridisciplinaire HAL, est destinée au dépôt et à la diffusion de documents scientifiques de niveau recherche, publiés ou non, émanant des établissements d'enseignement et de recherche français ou étrangers, des laboratoires publics ou privés. 


\title{
How to gain insight into the polydispersity of tannins: A combined MS and LC study
}

\author{
Laetitia Mouls*, Virginie Hugouvieux, Jean-Paul Mazauric, Nicolas Sommerer, Gérard Mazerolles, \\ Hélène Fulcrand
}

INRA, UMR1083, F-34060 Montpellier, France

Montpellier SupAgro, UMR1083, F-34060 Montpellier, France

Université Montpellier I, UMR1083, F-34060 Montpellier, France

\section{A R T I C L E I N F O}

\section{Article history:}

Received 13 January 2014

Received in revised form 16 May 2014

Accepted 21 May 2014

Available online 2 June 2014

\section{Keywords}

Tannin

Polyphenol

Polydispersity

Chromatography

Mass spectrometry

Polymer

\begin{abstract}
A B S T R A C T
In the context of the potential health benefits of food polyphenols, the bioavailability of tannins (i.e. proanthocyanidins) is a major issue, which is strongly influenced by the polydispersity and the degree of polymerisation of tannins. The average degree of polymerisation (DP) of tannins is usually determined using depolymerisation methods, which do not provide any information about their polymer distribution. Moreover, it is still a challenge to characterise tannin fractions of high polydispersity and/or containing polymers of high molecular weights, due to the limit of detection of direct mass spectrometry (MS) analysis methods. In the present work, the polydispersity of several tannin fractions is investigated by two complementary methods: a MALDI-MS method and a semi-preparative sub-fractionation. Using a combination of these methods we are able to gain insight into the DP distributions of the fractions consisting of tannins of medium and high DP. Moreover combining analyses can be useful to assess and compare the DP distributions of most tannin fractions.
\end{abstract}

(c) 2014 Elsevier Ltd. All rights reserved.

\section{Introduction}

Among phenolic compounds, tannins (Fig. 1) are widely distributed in the plant kingdom and consequently play a fundamental role in processed food. Nevertheless, their characterisation is still a challenge due to the heterogeneity in the degree of polymerisation (DP) of tannins. In a previous work, we underlined that (i) direct analysis (without chromatographic separation of the pure tannin sample) by MS enables the distribution of tannins of low DP to be determined and that (ii) detecting tannins composed of more than twenty-six monomeric units (DP26) is difficult. In this previous study (Mouls, Mazauric, Sommerer, Fulcrand, \& Mazerolles, 2011), the average DP (aDP) of four tannin fractions was estimated by chemical depolymerisation. A chemometric study of their mass spectra showed that they are strongly affected by the distribution of DPs in the fraction. Indeed, even under optimised conditions in electrospray ionisation mass spectrometry (ESI-MS), two fractions with similar aDP (as determined by chemical depolymerisation) generated different mass spectra

\footnotetext{
* Corresponding author at: INRA/Montpellier SupAgro, UMR1083, F-34060 Montpellier, France. Tel.: +33 (0)499 612 454; fax: +33 (0)499 612857 .

E-mail address: laetitia.mouls@supagro.inra.fr (L. Mouls).
}

(e.g., a fraction with a low polydispersity can have the same aDP as a fraction with a high polydispersity (Fig. S-1 in Supporting information) while two fractions with very different aDPs generated similar mass spectra due to the detection limit at DP26. To circumvent the difficulties of assessing the aDP by a direct MS analysis, we investigate in the present work the polydispersity of four tannin fractions using two complementary methods.

The first method relies on the MALDI-MS analysis of the soluble complexes formed between tannins and bovine serum albumin (BSA) (Mane et al., 2007). BSA is believed to make the ionisation of high DPs tannins easier, compared to direct MS (ESI-MS or MALDI-MS) analyses of tannins, and thereby to provide additional information on the DP distribution within the tannin fractions (Mane et al., 2007; Taylor, Barofsky, Kennedy, \& Deinzer, 2003; Yanagida, Kanda, Shoji, Ohnishi-Kameyama, \& Nagata, 1999).

The second method is based on the semi-preparative fractionation of the same four tannin fractions and the chemical depolymerisation (Rigaud, Perez-Ilzarbe, RicardodaSilva, \& Cheynier, 1991) of the collected sub-fractions. This procedure is expected to provide the aDP value of each tannin sub-fraction and give more details about the DP distribution of the four tannin fractions. The aDPs of the collected sub-fractions are also determined from the analyses by MALDI-MS of the tannin/BSA complexes. 


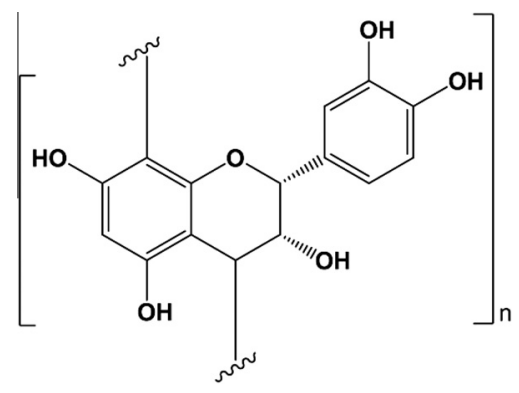

Fig. 1. Monomer unit (epicatechin) of the condensed tannins studied in this work.

\section{Experimental section}

\subsection{Preparation of the tannin fractions}

Fractions of tannins were prepared as described in the previous study (Mouls et al., 2011). They were obtained from two cider apple cultivars, Avrolles (A fractions) and Kermerrien (K fractions), in order to get fractions with various aDPs. Kermerrien cultivars are composed of tannins with aDP12 (Guyot, Doco, Souquet, Moutounet, \& Drilleau, 1997; Guyot, Marnet, Sanoner, \& Drilleau, 2003; Sanoner, Guyot, Marnet, Molle, \& Drilleau, 1999), whereas Avrolles cultivars exhibit significant amounts of tannins with aDP50. Freeze-dried powders of apple cortex tissues were prepared as described by Guyot et al. (2003). Extracts of tannins were firstly obtained using two consecutive solvent systems on each apple powder: methanol/acetic acid solution (99/1 v/v) (EK1 and EA1) then water/acetone/acetic acid solution (55.5/39.5/1 v/v/v) (EK2 and EA2). After concentrating methanol and water/acetone layers separately under vacuum, each extract was eluted using Fractogel ${ }^{\circledR}$ chromatography (Derdelinckx \& Jerumanis, 1984; Ricardo da Silva, Rigaud, Cheynier, Cheminat, \& Moutounet, 1991) (methyl acrylate copolymer in solution in $20 \%$ aqueous ethanol, HW-50F). The fractionation was achieved with methanol/acetic acid solution $(99 / 1 \mathrm{v} / \mathrm{v})$, then the highest molecular weights were eluted with a water/acetone/acetic acid solution (55.5/39.5/1 v/v/v). The fractions studied in this work are the last fractions (K1, K2, A1 and A2) eluted with water/acetone/acetic acid solution for each of the four extracts (EK1, EK2, EA1 and EA2).

\subsection{Chemical depolymerisation (thiolysis)}

A $1 \mathrm{mg} \mathrm{L}^{-1}$ solution of each dry extract was prepared in methanol. In a $250-\mu \mathrm{L}$ glass insert, $100 \mu \mathrm{L}$ of the solution were introduced and $100 \mu \mathrm{L}$ of toluene- $\alpha$-thiol (5\% v/v in acidified methanol with concentrated $0.2 \mathrm{~N} \mathrm{HCl}$ ) were added. After sealing the glass insert with an inert cap, reactions were carried out at $90^{\circ} \mathrm{C}$ for $2 \mathrm{~min}$.

The number aDP of tannin fractions was calculated from the depolymerisation reaction as the ratio between the summed molar concentrations of all constitutive units released as monomers and the summed molar concentrations of terminal constitutive units. Thiolysis of these fractions followed by reverse-phase HPLC analyses allowed their aDPs to be determined $\left(\mathrm{aDP}_{\mathrm{K} 1}=6.7\right.$, $\left.\mathrm{aDP}_{\mathrm{A} 1}=20.9, \mathrm{aDP}_{\mathrm{K} 2}=15.5, \mathrm{aDP}_{\mathrm{A} 2}=49.5\right)$.

\subsection{Analyses of tannin/BSA complexes by MALDI-MS}

Analyses of the tannin/BSA complexes were performed with an UltraFlex II MALDI-TOF/TOF (time-of-flight) mass spectrometer (Bruker Daltonics, Bremen, Germany). The mass spectra were acquired in linear mode over a mass range of $m / z 60,000-140,000$, corresponding to the $\mathrm{m} / \mathrm{z}$ range of tannin/protein complexes. Tannins were solubilised in water/acetic acid $(99 / 1, v / v)$ at a concentration of $9 \mathrm{mmol} \mathrm{L}^{-1}$ (the concentration is calculated using the aDP value of the fraction). For each tannin fraction, $5 \mu \mathrm{L}$ of the tannin solution were added to $1,495 \mu \mathrm{L}$ of a BSA solution at $30 \mu \mathrm{mol} \mathrm{L}^{-1}$ ( $45 \mathrm{nmol}$ of BSA and $45 \mathrm{nmol}$ of tannins). The 3,5-dimethoxy-4-hydroxycinnamic acid matrix solution at $10 \mathrm{mg} \mathrm{mL}^{-1}$ concentration in acetonitrile/water (50/50, v/v) acidified with $0.1 \%$ trifluoroacetic acid (TFA) was mixed with each equimolar tannin/BSA solution at a 50/50 (v/v) ratio. The doubledroplet spotting method was used: $1 \mu \mathrm{L}$ of the mixture was added to the MALDI target and after air-drying another $1 \mu \mathrm{L}$ was added. Mass calibration was performed externally; 800 laser shots were summed to obtain the final spectrum.

\subsection{Further fractionation of the tannin fractions}

Sub-fractionations of tannin fractions were run on a semipreparative HPLC (Gilson) fitted with a Develosil 100diol $5 \mu \mathrm{m}$ silica gel column (inner diameter $20 \mathrm{~mm}$, length $250 \mathrm{~mm}$; dr. Weber Consulting Ltd, Göd, Hungary). Twenty milligrams of each tannin fraction were dissolved in $1 \mathrm{~mL}$ of methanol and $1 \mathrm{~mL}$ of acetonitrile was added. The tannin solution was injected and separated by semi-preparative HPLC. The flow rate was $10 \mathrm{~mL} \mathrm{~min}^{-1}$ and the gradient conditions were solvent $\mathrm{A}$ (acetonitrile/acetic acid, 99.5/0.5, v/v); solvent B (methanol/water/acetic acid, 95.50/4/0.5, $\mathrm{v} / \mathrm{v} / \mathrm{v}$ ); initial 0\% B; 0-10 min, 30\% B linear; 10-35 min, 30\% B isocratic; $35-50 \mathrm{~min}, 50 \%$ B linear; $50-70 \mathrm{~min}, 50 \%$ B isocratic; 70-73 min, $80 \%$ B linear; $73-80 \mathrm{~min}, 80 \% \mathrm{~B}$ isocratic. The subfractions were manually collected at specific range times: subfraction 1 (s1): from $5 \mathrm{~min}$ to $20 \mathrm{~min}$; sub-fraction 2 (s2): from $20 \mathrm{~min}$ to $35 \mathrm{~min}$; sub-fraction 3 (s3): from $35 \mathrm{~min}$ to $50 \mathrm{~min}$; sub-fraction 4 (s4): from $50 \mathrm{~min}$ to $73 \mathrm{~min}$; sub-fraction 5 (s5): from $73 \mathrm{~min}$ to $88 \mathrm{~min}$. Each sub-fraction was concentrated under vacuum and freeze-dried.

\subsection{Data processing of the MALDI-MS spectra}

Mass spectra for BSA alone and tannins/BSA complexes were processed with Scilab software (Version 4.1.2). Data processing was performed on the same $\mathrm{m} / z$ range for all the mass spectra (between $m / z 60,000$ and 100,000). The tannin/BSA mass spectra were first aligned with the BSA mass spectrum. Then BSA and tannin/BSA mass spectra were normalised by signal intensity to the level of the BSA. The contribution of the residual free BSA from the tannin/BSA complexes was then subtracted. After this subtraction, the mass spectra were smoothed over 100 data points. Histograms showing the contributions of the different complexes to the MS spectrum were then constructed by taking the intensity at the theoretical $m / z$ value of (1:1) complexes of BSA with tannins of a given DP. From these histograms, the aDP can be calculated.

\section{Results and discussion}

3.1. Molecular weight distribution of the tannin fractions by MALDIMS analysis of the tannin/BSA complexes

ESI or MALDI mass spectra of tannin mixtures are always dominated by the lowest molecular weight species with peak intensities diminishing as the polymer chain length increases (Mane et al., 2007; Taylor et al., 2003; Yanagida et al., 1999). This limitation likely comes from ionisation issues of large size polymers, in such a way that tannins with DP higher than 26 cannot be detected under our conditions. This limitation has been overcome through the formation of tannin complexes by using bovine serum albumin (BSA) as a carrier. This approach enabled us to determine the DP distribution of tannin fractions containing 
polymers of higher DPs. However, experimental conditions have to be carefully selected in order to maximise the formation of stoichiometric tannin/BSA complexes and to keep all the complexes in solution. This implies the use of $1: 1$ tannins to protein molar ratio and the preparation of diluted complexes solutions. In the present work, solutions prepared with two tannin/protein molar ratios, typically 10:1 and 1:1, were compared. Using a 10:1 tannin/protein ratio the signal is broadened to high $m / z$ values and the aDP is overestimated, indicating the presence of non-stoichiometric tannin/protein complexes under these conditions. Afterwards, all the solutions were prepared with an equimolar mixture of tannins and protein. The soluble tannin/BSA complexes were analysed by MALDI-MS. They were detected as an unresolved "hump" above $m / z 67,000$, following the BSA signal between $m / z 65,000$ and 67,000 (Fig. 2). In the cases where tannins were added, we still observe a BSA peak, which reveals the presence of residual free BSA proteins. The different tannin fractions give rise to broad distributions of tannin/BSA complexes. Between $\mathrm{m} / \mathrm{z} 67,000$ and 73,000 , the $\mathrm{K} 1$ (red curve) and A1 (pink curve) mass spectra are superimposed and have a higher intensity than those obtained with K2 (blue curve) and A2 (green curve). This result indicates that, unlike the $\mathrm{K} 2$ and $\mathrm{A} 2$ fractions, the $\mathrm{K} 1$ and $\mathrm{A} 1$ fractions contain tannins with low molecular weights. After this strong excess of intensity for $m / z$ values just above the BSA peak, the A1 and $\mathrm{K} 1$ fractions show a rather sharp decrease for larger $m / z$ values. On the contrary, the A2 and $\mathrm{K} 2$ fractions show less intense signals close to the BSA peak and decrease more slowly at larger $m / z$. This can be seen in Fig. 2 where the intensity of each curve was normalised to the maximum of the corresponding residual BSA peak, thus emphasising the $m / z$ range of complexes. Note that while the relative intensities in a given spectrum reflect the abundance of the different ions, the comparison of the absolute intensities of different spectra is not relevant. Fig. 2 shows that the range of the $m / z$ values of the tannin/BSA complexes broadens at higher $m / z$ values with increasing aDPs (green curve). Note that the ion detected at around $m / z 80,000$ in the BSA mass spectrum may be a BSA fragment adduct or an impurity.

Subtracting the normalised BSA mass spectrum from the mass spectrum of the tannin/BSA complexes, as described in the experimental section, allows the DP distribution of each fraction to be estimated (Fig. 3). From the histograms, differences in the DP distributions are observed for the different tannin fractions (Fig. 3). The $\mathrm{K} 1$ and $\mathrm{A} 1$ fractions contain a large amount of tannins of low DPs (DP < 10), as compared to the K2 and A2 fractions. Regarding

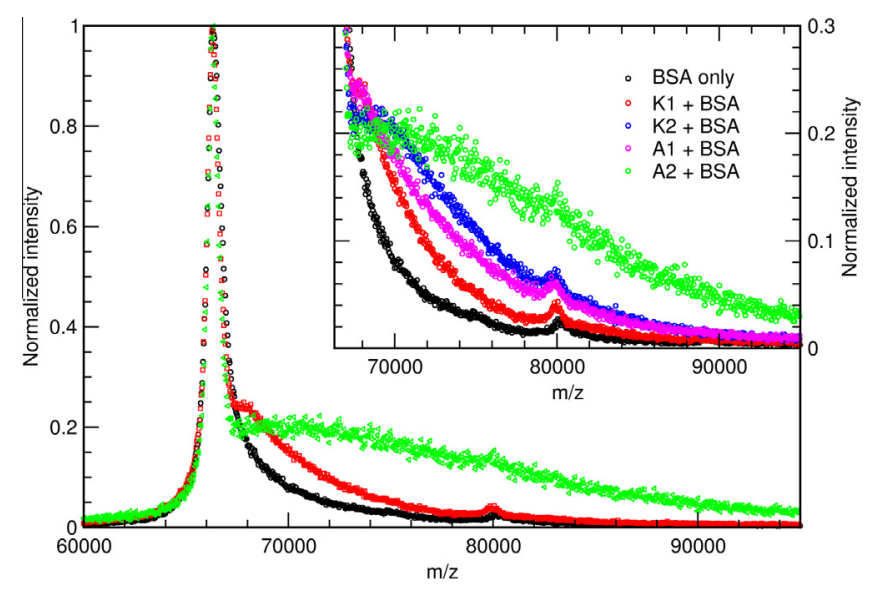

Fig. 2. Overlay of different MALDI-MS spectra: BSA alone and BSA in solution with the $\mathrm{K} 1$ or A2 tannin fraction. Inset: Details of the MALDI-MS spectra in the $m / z$ range corresponding to the tannin/BSA complexes; for each fraction, the intensity was normalised to the height of the corresponding free BSA peak.

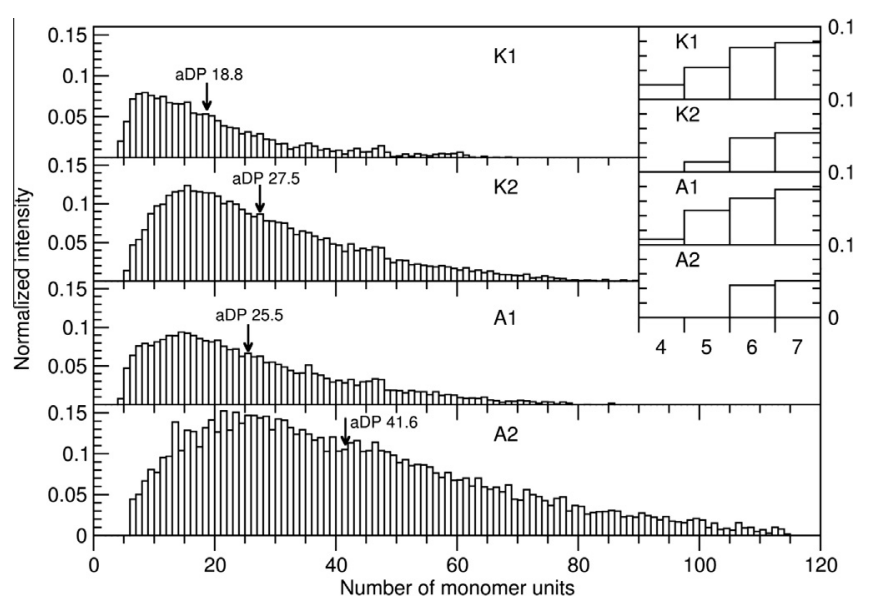

Fig. 3. Distribution of the number of monomer units per tannin/BSA complex for the four tannin fractions. The histograms are computed from the MALDI-TOF MS spectra shown in Fig. 2. The aDPs shown in Table 1 are calculated from the histograms. Inset: Abundance of the tannins of low DP in the four fractions.

the high DPs, the K2 and A1 tannin fractions have similar DP distributions; this is consistent with the aDP values determined by chemical depolymerisation (respectively aDP15.5 and aDP20.9). A closer look at the tannin distributions in the range from DP4 to DP7 (see inset in Fig. 3) shows two different trends: similar intensities are observed for the $\mathrm{K} 1$ and $\mathrm{A} 1$ fractions on one hand, and for the $\mathrm{K} 2$ and $\mathrm{A} 2$ fractions on the other hand. In the latter case, the lower DP tannins are weakly abundant or absent (no DP4 in the K2 and A2 fractions; DP5 only present in the K2 fraction but weakly abundant). Note that we were not able to distinguish the K1 and A1 fractions from ESI-MS analyses (aDP5.2 and aDP5.7 respectively from ESI-MS) although very different aDP values are determined by thiolysis (aDP6.7 and aDP20.9 respectively). This is due to the limitation of the direct MS analysis, which does not allow the detection of the tannins with high molecular weights present in the A1 tannin fraction (Fig. S-1 in Supporting information). These tannins with high DP are not taken into account in the aDP estimate and consequently the aDP determined from the ESI mass spectrum is underestimated.

The MALDI-MS method provides aDP values (Table 1) in good correlation with the values determined by thiolysis, except for the K1 tannin fraction that solely consists of low DPs. In fact, the aDP calculated for the latter is overestimated, which likely results from the ability of the BSA molecule to form complexes with several monomers and/or oligomers, hence favouring stoichiometries greater than $1: 1$ with tannins of low DP.

This MALDI-MS method is attractive as it provides quick information about the DP distribution of tannins, even for fractions containing tannins of high DP or very polydisperse. In the case of

Table 1

aDP determined by thiolysis, by ESI-MS and from MALDI mass spectra of tannin/BSA complexes.

\begin{tabular}{llll}
\hline \multirow{2}{*}{ Tannin fraction } & \multicolumn{2}{l}{ aDP } & \\
\cline { 2 - 4 } & Thiolysis & ESI-MS & MALDI-MS tannin/BSA \\
\hline K1 & 6.7 & 5.2 & 18.8 \\
K2 & 15.5 & 11.1 & 27.5 \\
A1 & 20.9 & 5.7 & 25.5 \\
A2 & 49.5 & 11.8 & 41.6 \\
A1-s5 & 61.4 & No signal & 46.4 \\
A2-s5 & 96.8 & No signal & 62.9 \\
\hline
\end{tabular}


tannin fractions consisting only of low DP tannins, this method should nevertheless be combined to a direct analysis by ESI-MS because the latter is more accurate for estimating the polydispersity of this type of fractions. Hence, this study shows that it is possible to estimate the polydispersity of tannin fractions by MS through a combination of the MALDI-MS analysis of tannin/BSA complexes and direct analysis of tannins by MS.

In the following, we obtained more knowledge on the fraction composition by performing a further fractionation on a semi-preparative HPLC system. Then, aDP estimates of the resulting sub-fractions were determined by chemical depolymerisation (thiolysis) and compared to the MALDI-MS results.

\subsection{Semi-preparative HPLC fractionation}

Semi-preparative HPLC followed by depolymerisation analysis of the resulting sub-fractions was performed, to determine the DP distributions of tannins in the original fractions. Five subfractions were collected manually at specific range times (Fig. 4). The tannins corresponding to sub-fractions s1, s2, s3 are oligomers ranging from DP1 to DP14, which can be detected by direct MS analyses. The tannins collected in sub-fractions s4 were eluted as an unresolved broad peak (first "hump") and only tannins at the beginning of the peak can be detected by ESI-MS (up to DP20-23 depending on the initial tannin fractions). The tannins of higher DPs, eluted with $80 \%$ of methanol (solvent B) in the last part of the gradient as another broad peak (second "hump") and collected in sub-fractions s5 cannot be detected by direct MS analyses (data not shown).

After concentration and freeze-drying, the aDP value of each sub-fraction was determined by chemical depolymerisation (Fig. 4). Based on the chromatographic profiles and the aDP values of the sub-fractions, we can see that the $\mathrm{K} 1$ fraction mainly contains tannins of low DPs. The K2 and A1 fractions show similar profiles up to medium DPs but A1 contains much more tannins of high DPs (collected in s5, aDP61.4) than K2 (s5 with aDP23.9). The A2 fraction rather shows a different profile, with a weak UV signal in the first three sub-fractions, and a large amount of tannins in s4 and s5 (with aDP27.1 and aDP96.8 respectively). A more quantitative comparison of the DP composition of the fractions can be performed by pooling the results obtained for sub-fractions with close aDPs. Thus, we defined six classes of aDPs: aDP5, aDP10, aDP20, aDP27, aDP61 and aDP97. For example, sub-fractions s1 and s2 of fractions K1, K2 and A1 belong to the aDP5 class. For each fraction, we calculated the molar percentage of tannins in each class of aDP (Fig. 5), using the average molecular weight (aMW) and the mass of lyophilised tannins from each sub-fraction (Table S-1 in Supporting information).

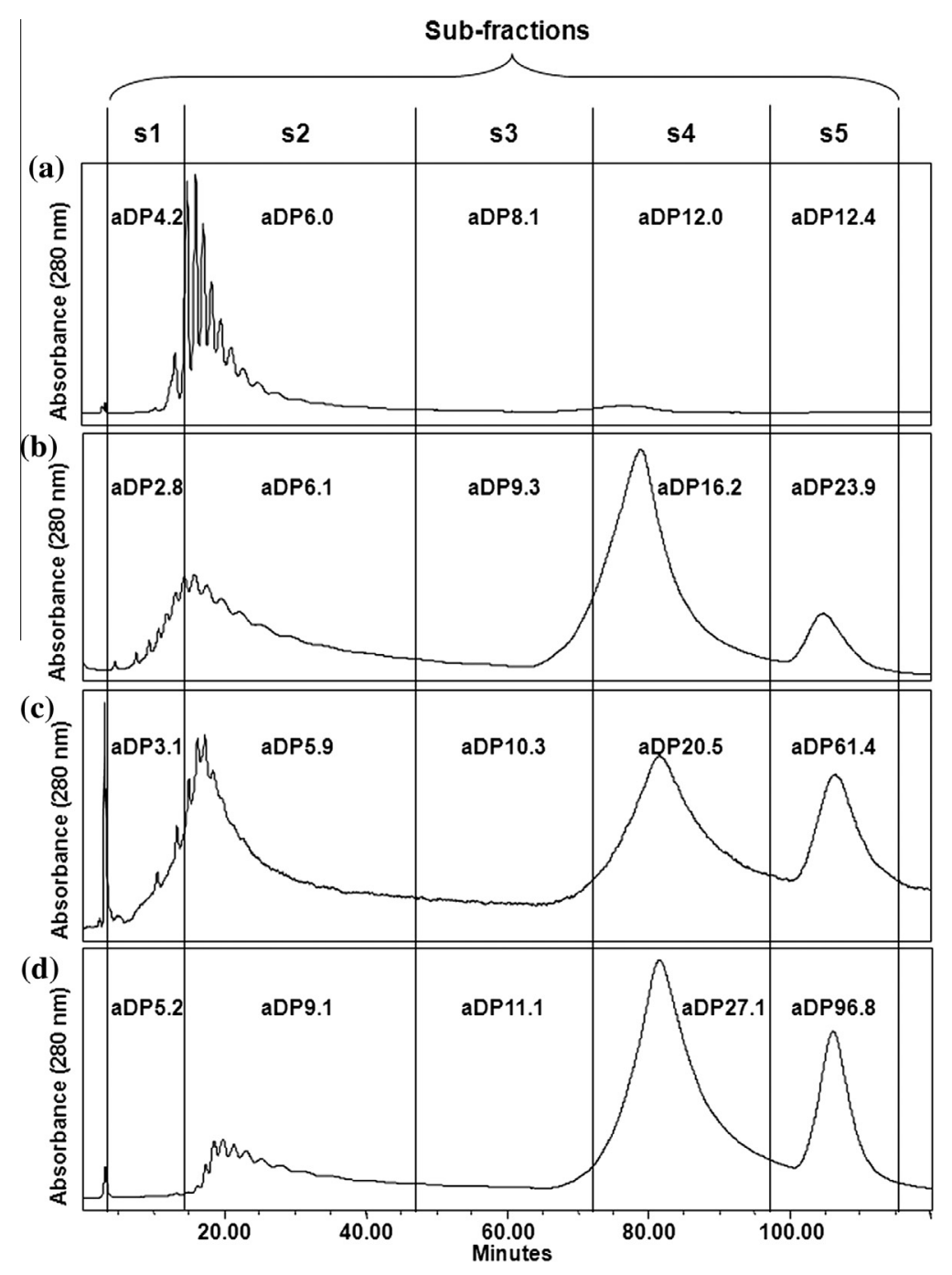

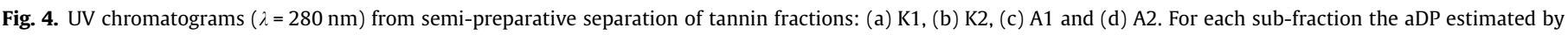
thiolysis is indicated. 


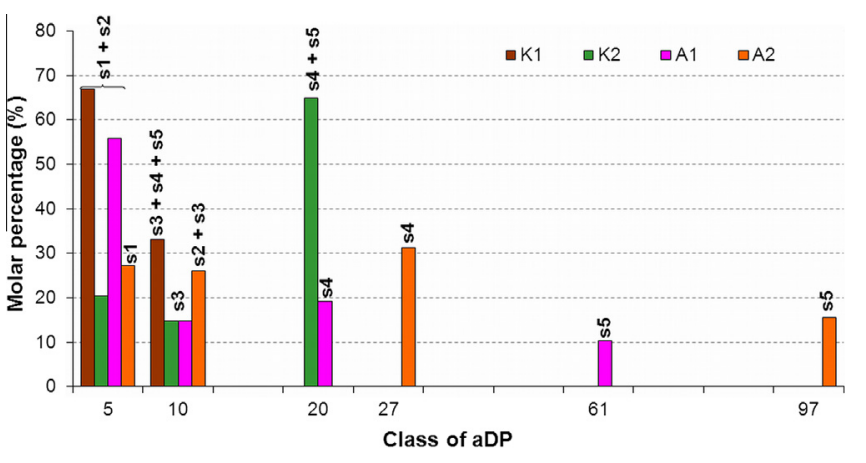

Fig. 5. Distribution of tannin DPs within the $K 1, K 2, A 1$ and $A 2$ fractions. The proportions of the different aDP classes (aDP5, aDP10, aDP20, aDP27 aDP61 and aDP97) expressed in molar percentages were calculated by pooling the results of sub-fractions with similar aDPs.

Fig. 5 shows the composition of the four initial fractions $\mathrm{K} 1, \mathrm{~K} 2$, A1 and A2, expressed as molar percentage of the aDP classes. These histograms emphasise the molecular weight distribution of tannins. Low DP tannins are the most abundant classes $(\mathrm{aDP} \leqslant 10)$ in the $\mathrm{K} 1$ and $\mathrm{A} 1$ tannin fractions (accounting for $100 \%$ and $70.6 \%$ respectively), whereas $\mathrm{K} 2$ is mainly composed of tannins of the aDP20 class (65.0\%). For A2, almost half of the tannins are distributed in aDP27 (31.3\%) and aDP97 (15.5\%) classes. The large presence of low DP tannins only in fractions $\mathrm{K} 1$ and $\mathrm{A} 1$ is in accordance with results given by direct mass spectrometry analyses and by MALDI-MS analysis of the complexes.

Indeed, only the lowest DP tannin ions of the K1 and A1 fractions are detected in the ESI-MS analyses. However, the A1 fraction contains a proportion of tannins with $\mathrm{aDP} \leqslant 10$ much higher (70.6\%) than the proportion of tannins with aDP20 (19.1\%). The non-detection of the highest DP tannins in A1 can be attributed to the decrease in tannin ionisation with increasing molecular weights, which leads to a limit of detection for DP higher than 26 (Mouls et al., 2011).

Conversely, the $\mathrm{K} 2$ fraction is composed of a majority of tannins containing 20 monomer units on average, with, furthermore, a narrow distribution of the DP values. This leads to a good correlation between the aDP calculated from the ESI mass spectrum and the aDP obtained by depolymerisation. Thus, tannins of higher DP are detected only if tannins of lower DP are weakly abundant or absent. The A1 fraction (aDP20.9) contains a very large range of tannin DPs, contrarily to the K2 fraction (aDP15.5) that is centred on the aDP value. The A1 and K2 tannin fractions which have similar aDP (respectively aDP27.5 and aDP25.5 from the MALDI-MS analyses) give distinct ESI mass spectra (Fig. S-1 in Supporting information) because the A1 tannin fraction is more polydisperse than the K2 tannin fraction. The A1 tannin fraction is composed of low DP tannins detected by MS and of high DP tannins which are not detected by direct MS analysis and distort the aDP estimate. The same phenomenon is observed for the $\mathrm{K} 2$ and $\mathrm{A} 2$ tannin fractions; they have a similar ESI mass spectrum profile because the $\mathrm{A} 2$ fraction is composed of very large tannins which are not detectable by ESI mass spectrometry (15.5\% of aDP97 tannins and $31.3 \%$ of aDP27).

These results strengthen the idea that the presence of low DP tannins (from DP1 to DP9) hinders the detection of larger DP on the mass spectrum. Thus, the direct analysis of tannin fractions by ESI mass spectrometry can be all the more deceptive, as the detection is limited to tannins with DP values lower than 26. With the use of tannin/BSA complexes, ionisation of the high DP tannins (complexed with BSA) becomes possible and it is the reason why the use of tannin/BSA complexes is much more efficient for estimating the polydispersity of tannin fractions with high DP by mass spectrometry.

\section{3. aDP estimation of sub-fractions from tannin/BSA complexes mass spectrum}

The fractionation procedure revealed the presence of highly polymerised tannin sub-fractions s5 in the A1 and A2 fractions. These sub-fractions were analysed using the MALDI-MS method. With respect to the sub-fractions with the highest aDPs, A1-s5 and A2-s5 (thiolysis aDP values: aDP61.4 and aDP96.8, respectively), the aDP values from the MALDI-MS method are underestimated (respectively aDP46.4 and aDP62.9). Numerous studies have shown that MALDI-MS analyses underestimate the compounds with high molecular weights, which may be caused by sample preparation (Schriemer \& Li, 1997), discrimination in ion detection (Larsen, Simonsick, \& McEwen, 1996; Tang, Dreifuss, \& Vertes, 1995) and sensitivity to laser power (Martin, Spickermann, Rader, \& Mullen, 1996). This effect of spectral discrimination previously shown in MALDI time-of-flight mass spectrometry for polydisperse synthetic polymers is thus also observed in the present study for the very high DP tannins; however these results are interesting because it is the first time that aDP values as high as this can be deduced from an MS analysis. Furthermore, the aDP values obtained by chemical depolymerisation are not necessarily accurate because this method has its own limitations and may also distort the estimate of the aDP. Indeed, failure to recover all the terminal units of tannins after thiolysis leads to overestimates of the aDP. Besides, tannins may undergo a variety of chemical modifications, due to their chemical reactivity and specifically their high reducing properties. These chemical changes are not accounted for in the calculation of the aDP from thiolysis. It is consequently difficult to know which of the two aDP values estimated by MALDI-MS method or thiolysis is closest to the actual aDP value. The MS method has the advantage of stretching the detection of tannins to higher DP compared to the direct MS analysis, with an aDP limit value of around 64. By the way, a few varieties (such as cider apple (Sanoner et al., 1999) or cashew apple (Michodjehoun-Mestres et al., 2009)) synthesise very high DP tannins (above aDP 60), which are generally discarded during food-processing (precipitation with other macromolecules or interaction with equipment during filtrations). Consequently this MALDI-MS method can give insight into the polydispersity of condensed tannins in most products. It is moreover the only MS method that allows the determination of aDP values of tannin fractions containing high DP polymers correlated with the aDP estimated by thiolysis. Indeed, the combination of MALDIMS on complexes and thiolysis will give access to a more accurate estimate of the polydispersity of tannin fractions.

From this work we can propose some guidelines for determining the aDP and polydispersity of tannin fractions. The first step consists in calculating the aDP from chemical depolymerisation. If the aDP is lower than 15 the second step will consist in running direct MS analysis (either ESI or MALDI). If the values determined by thiolysis and ESI-MS analysis are similar, the DP distribution of the tannin fraction is narrow. If the fraction contains a wide range of DPs, further MS analyses using tannin/protein complexes are required. If the aDP determined by thiolysis is higher than 15 , the MALDI-MS method will give a better estimate of the aDP and give insight into the polydispersity. Above aDP50 and for increasing values of the aDP as determined by thiolysis, the aDP values determined with the MALDI method increase as well; however, the discrepancy between thiolysis and MALDI increases (see Table 1). Lastly, if the tannin fraction is composed of very high DP tannins it will be more difficult to estimate accurately the aDP value, owing to the respective limits of the methods. Fractionation will be helpful in this case. 


\section{Conclusions}

The work reported here reveals the significant and non-trivial influence of the polydispersity of tannin fractions on the mass spectrum profiles.

A combination of direct ESI-MS analyses of tannins and MALDI MS analyses of tannin/BSA complexes enables the polymer distribution of most tannin fractions to be characterised. By using various techniques, the present study emphasises the major influence of the polydispersity of tannin fractions on the mass spectrometry ionisation response. The MALDI-MS method takes advantage of the tannins' property to interact with proteins and it is an attractive alternative to estimate the DP distribution of tannins. Using this MALDI-MS method a sensitive improvement of the aDP estimates is obtained for the tannin fractions with medium and high DPs, with calculated aDP up to aDP62. For this kind of fraction, the aDPs estimated by using the complexation of BSA and tannins are much closer to the values determined by thiolysis, as compared to the aDPs determined by direct MS analysis.

Eventually more accurate estimates of the polydispersity of tannin fractions will be useful in numerous food processes and in nutrition. For example, determining the relationship between the degree of polymerisation of tannins and their bioavailability would be a major step forward towards the understanding of the health effects of tannins.

The present work on tannin polydispersity intends to complement the previously published chemometric study. Together these two studies have used a range of techniques (direct analysis by MS, MALDI-MS method, fractionation and depolymerisation methods, chemometrics) and give a comprehensive view of the behaviour of tannins in mass spectrometry. They provide a guideline procedure for a more complete study of condensed tannins.

\section{Acknowledgement}

The authors would like to thank Dr Sylvain Guyot for providing freeze-dried powder of apple cortex tissues.

\section{Appendix A. Supplementary data}

Supplementary data associated with this article can be found, in the online version, at http://dx.doi.org/10.1016/j.foodchem.2014. 05.121 .

\section{References}

Derdelinckx, G., \& Jerumanis, J. (1984). Separation of malt hop proanthocyanidins on Fractogel TSK HW-40 (S). Journal of Chromatography, 285, 231-234.

Guyot, S., Doco, T., Souquet, J. M., Moutounet, M., \& Drilleau, J. F. (1997). Characterization of highly polymerized procyanidins in cider apple (Malus sylvestris var. Kermerrien) skin and pulp. Phytochemistry, 44(2), 351-357.

Guyot, S., Marnet, N., Sanoner, P., \& Drilleau, J.-F. (2003). Variability of the polyphenolic composition of cider apple (Malus domestica) fruits and juices. Journal of Agricultural and Food Chemistry, 51(21), 6240-6247.

Larsen, B. S., Simonsick, W. J., \& McEwen, C. N. (1996). Fundamentals of the application of matrix-assisted laser desorption ionization mass spectrometry to low mass poly(methylmethacrylate) polymers. Journal of the American Society for Mass Spectrometry, 7(3), 287-292.

Mane, C., Sommerer, N., Yalcin, T., Cheynier, V., Cole, R. B., \& Fulcrand, H. (2007). Assessment of the molecular weight distribution of tannin fractions through MALDI-TOF MS analysis of protein-tannin complexes. Analytical Chemistry, 79(6), 2239-2248.

Martin, K., Spickermann, J., Rader, H. J., \& Mullen, K. (1996). Why does matrixassisted laser desorption/ionization time-of-flight mass spectrometry give incorrect results for broad polymer distributions? Rapid Communications in Mass Spectrometry, 10(12), 1471-1474.

Michodjehoun-Mestres, L., Souquet, J.-M., Fulcrand, H., Meudec, E., Reynes, M., \& Brillouet, J.-M. (2009). Characterisation of highly polymerised prodelphinidins from skin and flesh of four cashew apple (Anacardium occidentale L.) genotypes. Food Chemistry, 114(3), 989-995.

Mouls, L., Mazauric, J.-P., Sommerer, N., Fulcrand, H., \& Mazerolles, G. (2011) Comprehensive study of condensed tannins by ESI mass spectrometry: Average degree of polymerisation and polymer distribution determination from mass spectra. Analytical and Bioanalytical Chemistry, 400(2), 613-623.

Ricardo da Silva, J. M., Rigaud, J., Cheynier, V., Cheminat, A., \& Moutounet, M. (1991). Procyanidin dimers and trimers from grape seeds. Phytochemistry, 30(4), 1259-1264.

Rigaud, J., Perez-Ilzarbe, X., RicardodaSilva, J. M., \& Cheynier, V. (1991). Micro method for the identification of proanthocyanidin using thiolysis monitored by high-performance liquid chromatography. Journal of Chromatography, 540, 401-405.

Sanoner, P., Guyot, S., Marnet, N., Molle, D., \& Drilleau, J. (1999). Polyphenol profiles of French cider apple varieties (Malus domestica sp.). Journal of Agricultural and Food Chemistry, 47(12), 4847-4853.

Schriemer, D. C. \& Li, L. (1997). Mass discrimination in the analysis of polydisperse polymers by MALDI time-of-flight mass spectrometry. 1. Sample preparation and desorption/ionization issues. Analytical Chemistry, 69(20), 4169-4175.

Tang, X. D., Dreifuss, P. A., \& Vertes, A. (1995). New matrices and accelerating voltage effects in matrix-assisted laser-desorption ionization of syntheticpolymers. Rapid Communications in Mass Spectrometry, 9(12), 1141-1147.

Taylor, A. W., Barofsky, E., Kennedy, J. A., \& Deinzer, M. L. (2003). Hop (Humulus lupulus L.) proanthocyanidins characterized by mass spectrometry, acid catalysis, and gel permeation chromatography. Journal of Agricultural and Food Chemistry, 51(14), 4101-4110.

Yanagida, A., Kanda, T., Shoji, T., Ohnishi-Kameyama, M., \& Nagata, T. (1999). Fractionation of apple procyanidins by size-exclusion chromatography. Journal of Chromatography A, 855(1), 181-190. 